\title{
De novo inflammatory bowel disease is a potential post-acute sequela of SARS-CoV-2 infection
}

\author{
Thanita Thongtan MD*, Anasua Deb MD*, Sameer Islam MD
}

\begin{abstract}
Background: Even though patients with inflammatory bowel disease (IBD) are not at increased risk of COVID-19 infection, patients with post-acute COVID-19 have been reported to have de novo IBD or a new diagnosis of IBD.

Objective: This article reviews the presentation, diagnosis, and clinical course of patients described in the literature to have new-onset IBD after the diagnosis of COVID-19 infection and discusses the possible pathophysiological mechanism.

Methods: Extensive literature review by compiling information from case reports and original studies identified by a PubMed and EMBASE search from inception to May 2021.

Results: We identified 4 case reports de novo IBD that occurred 2 weeks to 5 months after acute COVID-19 infection. Patients presented with persistent bloody diarrhea, abdominal pain, and anemia. Three patients were diagnosed with ulcerative colitis, and one patient was diagnosed with Crohn's disease. Available evidence suggests that COVID-19 infection can cause intestinal inflammation and trigger de novo IBD, potentially through intestinal barrier leakage, alterations in gene expression, gut microbiota dysbiosis, and an exaggerated immune response.

Conclusion: The presence of the SARS-CoV-2 virus in the gut can cause de novo IBD through complex multiple factors. Further studies need to be done to confirm a causal link and the underlying mechanism. Clinicians should be vigilant about the possibility of IBD in patients present with anemia, abdominal pain, or chronic bloody diarrhea after a short interval of COVID-19 infection which may warrant a referral to a gastroenterologist.
\end{abstract}

Keyword: de novo inflammatory bowel disease, COVID-19 infection, SARS-CoV-2 virus, pathophysiology

\section{INTRODUCTION}

In December 2019, viral infections cause by severe acute respiratory syndrome coronavirus 2 (SARSCoV-2) emerged as a global pandemic. ${ }^{1}$ Although most patients infected with SARS-CoV-2 have respiratory tract involvement, it is now clear that the virus can infect any organ, including the gastrointestinal system $^{2}$ as

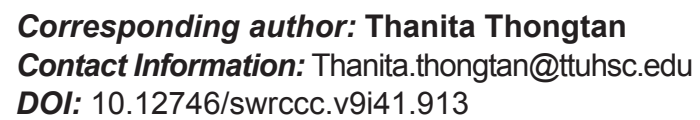

the viral host receptor, angiotensin-converting enzyme (ACE) 2, is distributed not only in the lung but also in the gastrointestinal tract. ${ }^{3}$ Even though patients with inflammatory bowel disease (IBD) are not at increased risk of COVID-19 infection, ${ }^{4}$ there have been reports on patients who developed de novo IBD as a post-acute sequela of SARS-CoV-2 infection. ${ }^{5-8}$ The mechanisms underlying both $\mathrm{IBD}^{9}$ and SARS-CoV-2 infection ${ }^{10}$ pathophysiology are not completely understood. This study reviews the current evidence on the potential pathophysiology behind the emergence of de novo IBD following acute SARS-CoV-2 infection.

\footnotetext{
* Co-first author.
} 


\section{Methods}

We searched PubMed and EMBASE using the search term of "(inflammatory bowel disease [MeSH Terms]) AND ((covid-19) OR (coronavirus) OR (SARSCoV-2))" from inception to April 2021, to identify all studies reporting the new-onset IBD in patients with a history of COVID-19 infection. We also searched for current evidence on the mechanisms underlying gastrointestinal involvement in COVID-19 infection and inflammatory bowel disease to understand the potential link between the two. We excluded studies that reported findings in patients with established diagnosis of IBD. The initial search yielded 1,243 articles that were relevant (965 articles from EMBASE and 278 studies from PubMed).

\section{Case summaries}

We identified four articles reported cases of de novo IBD that were diagnosed ranging from 2 weeks to 5 months after acute COVID-19 infection (Table 1). The mean age of diagnosis was 30 years, $50 \%$ were female. Common presentations included bloody diarrhea, abdominal pain, and anemia. Three patients were diagnosed with ulcerative colitis, and one patient was diagnosed with Crohn's disease. All patients met the criteria for the diagnosis of IBD by using a combination of clinical features and characteristic findings on endoscopy, as well as histopathologic analysis. SARSCoV-2 was found to be negative in two cases's intestinal samples. ${ }^{5,8}$ Two cases were reported improvement with IBD treatment. ${ }^{6,8}$

\section{INSIGHTS FROM MOLECULAR BIOLOGY}

Various components of the renin-angiotensin system (RAS), including ACE2, are expressed in the terminal ileum and colonic mucosa. ${ }^{11}$ The expression and activity of ACE2 were found to be lower in a colonic biopsy from IBD patients with active disease as compared to IBD patients with no inflammation. ${ }^{12}$ Microarray transcriptomics for expressed RNA from colonic biopsies of IBD patients have shown that ACE2 transcript copies were $70 \%$ more in UC and $30 \%$ more in CD compared to controls $;{ }^{13}$ however, the results need to be interpreted with caution since RNA expression does not always reflect in protein expression. In the small bowel biopsies of Crohn's disease patients, on the other hand, the expression of ACE2 was reduced..$^{14}$ In animal studies, ACE2 knockout predisposes to increased susceptibility to colitis through mechanisms involving altered gut microbiota and increased expression of the proinflammatory molecule angiotensin II. ACE2 knockout was also found to impede the absorption of tryptophan, the precursor to niacin, the deficiency of which has been linked to intestinal inflammation. ${ }^{15}$ In fact, patients with IBD, particularly Crohn's disease, have lower serum tryptophan levels compared to control patients with non-IBD intestinal inflammation. ${ }^{16}$

SARS-CoV-2 through its interaction with ACE2 receptors on target mucosal membranes triggers downstream effectors that perpetuate inflammatory response with increased fecal calprotectin and serum IL-6 expression. ${ }^{17}$ SARS-CoV-2 binding to the ACE2 receptors causes their internalization resulting in downregulation of the surface expression of ACE2, ${ }^{18-20}$ which in itself, can predispose to a pro-inflammatory environment through mechanisms discussed above. SARS-CoV-2 binding to ACE2 receptors results in tryptophan deficiency, reduced angiotensin 1-7 levels, and elevated angiotensin II and tumor necrosis factoralpha expression, ${ }^{21}$ all of which trigger inflammation in the gut.

Transmembrane serine protease (TMPRSS) -2 and -4 are another class of membrane protein in the colonic mucosa, which primes the SARS-CoV-2 spike protein, allowing it to bind more efficiently to ACE2. ${ }^{22-24}$ Analysis of autopsy tissue from fatal COVID-19 cases showed that SARS-CoV-2 RNA was highly colocalized in cells expressing TMPRSS2. ${ }^{25}$ Interestingly, microarray analysis for RNA expression of ileal TMPRSS2 was $30 \%$ higher in the ileum of patients with UC as compared to controls. ${ }^{13,26}$

Besides ACE2 and TMPRSS2, several other genes, particularly those involved in innate immune signaling, are upregulated in IBD and may contribute to the pro-inflammatory state in the gut. Suarez-Farinas et al. studied the gene expression in SARS-CoV-2 infection and compared that to the genes which are differentially upregulated in inflamed regions of the gut 
Table 1. Clinical Summaries of Case Reports

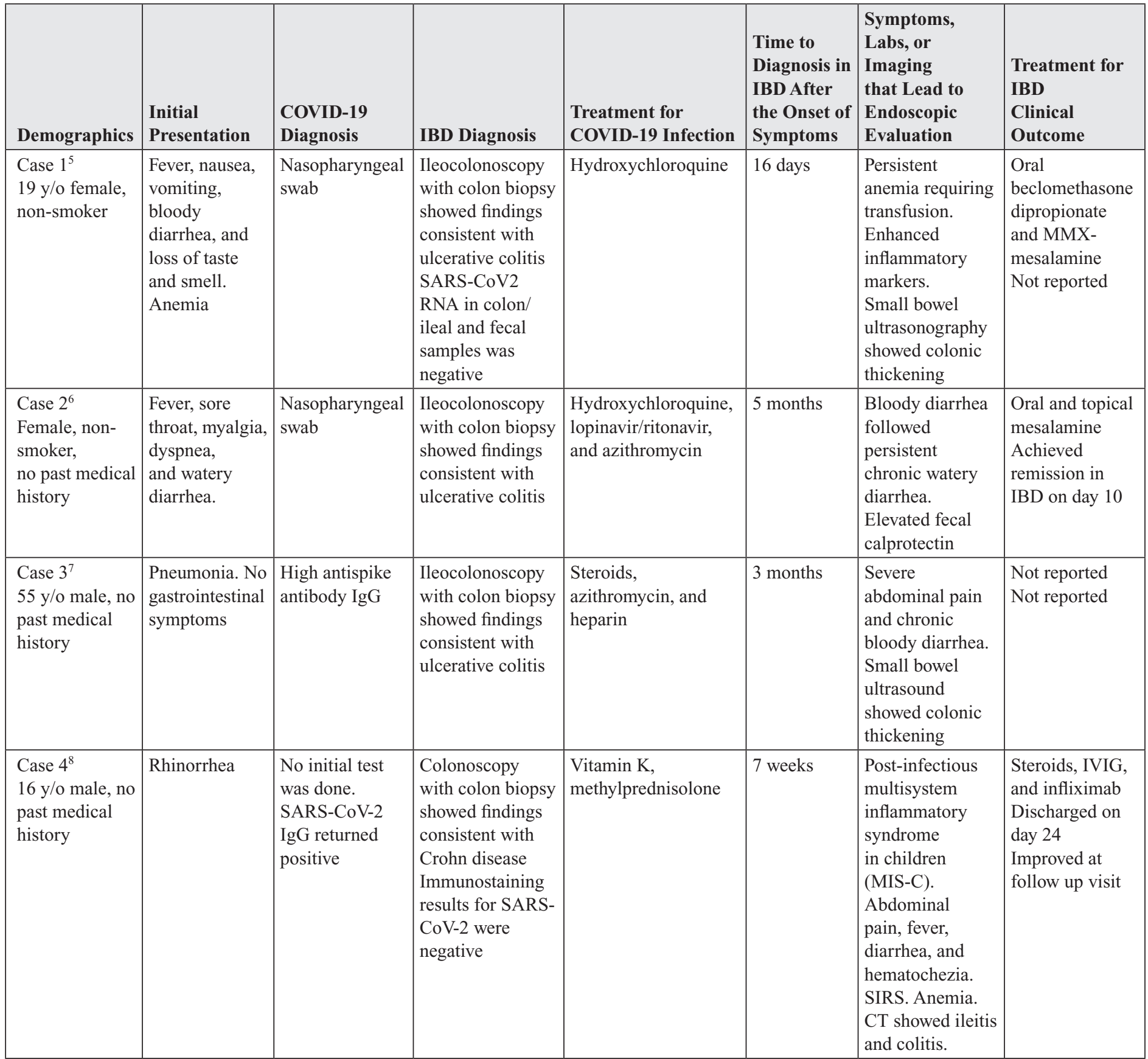

in IBD. ${ }^{26}$ They also studied the genes that are upregulated in the peripheral blood in patients with active IBD and compared that to genes up-regulated in the blood of COVID-19-infected patients. Using both these approaches, the authors found that there is a significant overlap of the genes that are over-expressed in active IBD as well as COVID-19 infection. These genes include IL6, CXCL1/2/5, PDPN, and S100A8/A9. Several other genes that are relevant for IBD pathways were also upregulated with SARS-CoV-2 infection of murine models such as dextran sodium sulfate (DSS), TNBS, and T-cell transfer colitis model. All these 
upregulated genes contribute towards an environment of exaggerated immune response in the gut which is one of the characteristics of IBD pathogenesis. ${ }^{9}$

Another crucial pathophysiologic mechanism for IBD that has been explored in detail is the dysbiosis of the gut microbiota. ${ }^{27-29}$ Such dysbiosis may either deplete the anti-inflammatory microbial metabolites (e.g., acetate, propionate, and butyrate group of shortchain fatty acids) or increase the amount of proinflammatory metabolites. ${ }^{30}$ COVID-19 infection has also been linked to similar effects in the gut, in that SARS-COV-2 infection promotes alteration in the gut microbiota leading to dysbiosis. ${ }^{31,32}$ Real-time analysis of the fecal microbiota in hospitalized patients with COVID-19 infection showed that the infection led to persistent alteration of the gut microbiome as compared to uninfected controls, resulting in deletion of beneficial commensals and enrichment of potential pathogens. ${ }^{32}$ Such alteration in the gut microbiome can lead to altered immune pathway regulation and increased expression of inflammatory pathways.

Post-COVID conditions associated with a systemic inflammatory reaction, including gastrointestinal damage, were driven by uncontrolled immune system activation and resulted in cytokine storm. ${ }^{33}$ Initial plasma IL1B, IL1RA, IL7, IL8, IL9, IL10, basic FGF, GCSF, GMCSF, IFN $\gamma$, IP10, MCP1, MIP1A, MIP1B, PDGF, TNF $\alpha$, and VEGF concentrations were higher in the patients with COVID-19 infection than in healthy adults suggesting higher expression of T-helper-1 cell response. ${ }^{1}$ Similarly, the excessive mucosal immune responses against food antigens and commensal microbiota through an increase in T-helper-1 cells, T-helper-17 cells, and regulatory T cells may contribute to the pathogenesis of IBD. ${ }^{9}$

\section{Discussion}

Available evidence suggests that COVID-19 infection can cause intestinal inflammation and trigger de novo IBD, potentially through intestinal barrier leakage, changes in genetic expression, gut microbiota dysbiosis, and excessive immune responses. It should be noted that all reported cases developed de novo IBD weeks to months following acute SARS-CoV-2 infection and not during the acute phase. Because of the large number of COVID-19 infections worldwide, these two events could have occurred in close proximity coincidentally or causally. To definitively establish a causal link, a larger number of COVID19 patients developing IBD needs to be studied, and more research into the underlying mechanism needs to be conducted. Gene expression and epigenetic modifications of the inflamed gastrointestinal tissues from the patients with post-COVID-19 de novo IBD should be tested in order to understand the molecular pathways involved in the pathogenesis as well as the distinguished subtypes of the IBD. Clinicians should be vigilant about the possibility of IBD in patients with new-onset anemia, abdominal pain, or chronic bloody diarrhea following COVID-19 infection, and this may warrant a referral to a gastroenterologist.

Article citation: Thongtan T, Deb A, Islam S. De novo inflammatory bowel disease is a potential post-acute sequela of SARS-CoV-2 infection The Southwest Respiratory and Critical Care Chronicles 2021;9(41):35-39 From: Department of Internal Medicine, Texas Tech University Health Sciences Center, Lubbock, Texas Submitted: $7 / 18 / 2021$

Accepted: 9/26/2021

Reviewer: Drew Payne DO

Conflicts of interest: none

This work is licensed under a Creative Commons Attribution-ShareAlike 4.0 International License.

\section{REFERENCES}

1. Huang C, Wang Y, Li X, et al. Clinical features of patients infected with 2019 novel coronavirus in Wuhan, China. Lancet (London, England) $2020 \mathrm{Feb}$;395(10223):497-506.

2. Ma C, Cong Y, Zhang H. COVID-19, and the Digestive System. Am J Gastroenterol [Internet]. 2020;115(7):1003-6.

3. Xiao F, Tang M, Zheng X, et al. Evidence for Gastrointestinal infection of SARS-CoV-2. Gastroenterology [Internet] 2020;158(6):1831-1833.e3. Available from: https://doi.org/ $10.1053 /$ j.gastro.2020.02.055

4. Taxonera C, Sagastagoitia I, Alba C, et al. 2019 novel coronavirus disease (COVID-19) in patients with inflammatory bowel diseases. Aliment Pharmacol Ther 2020 Jul;52(2):276-83.

5. Calabrese E, Zorzi F, Monteleone G, et al. Onset of ulcerative colitis during SARS-CoV-2 infection. Dig Liver Dis [Internet] 2020/06/11. 2020 Nov;52(11):1228-9. 
6. Taxonera C, Fisac J, Alba C. Can COVID-19 trigger de novo inflammatory bowel disease? Gastroenterology 2021 Mar; 160(4):1029-30.

7. Imperatore N, Bennato R, D'Avino A, et al. SARS-CoV-2 as a trigger for de novo ulcerative colitis. Inflamm Bowel Dis [Internet] 2021 Feb 22; Available from: https://doi.org/ 10.1093/ibd/izab040

8. Sweeny KF, Zhang YJ, Crume B, et al. Inflammatory bowel disease presenting with concurrent covid-19 multisystem inflammatory syndrome. Pediatrics 2021;147(4): e2020027763.

9. Chang JT. Pathophysiology of inflammatory bowel diseases. N Engl J Med [Internet] 2020 Dec 30;383(27):2652-64.

10. Yuki K, Fujiogi M, Koutsogiannaki S. COVID-19 pathophysiology: A review. Clin Immunol [Internet] 2020/04/20. 2020 Jun;215: 108427. Available from: https://pubmed.ncbi. nlm.nih.gov/32325252

11. Garg M, Royce SG, Lubel JS. Letter: intestinal inflammation, COVID-19 and gastrointestinal ACE2 - exploring RAS inhibitors. Aliment Pharmacol Ther 2020;52(3):569-570.

12. Garg M, Royce SG, Tikellis $C$, et al. Imbalance of the renin-angiotensin system may contribute to inflammation and fibrosis in IBD: A novel therapeutic target? Gut 2020;69(5): 841-854.

13. Nowak JK, Lindstrøm JC, Kalla R, et al. Age, inflammation, and disease location are critical determinants of intestinal expression of SARS-CoV-2 Receptor ACE2 and TMPRSS2 in inflammatory bowel disease. Gastroenterology 2020;159(3):1151-1154.

14. Potdar AA, Dube S, Naito T, et al. Altered intestinal ACE2 levels are associated with inflammation, severe disease, and response to anti-cytokine therapy in inflammatory bowel disease. Gastroenterology 2021;160(3):809-822.

15. Perlot T, Penninger JM. ACE2 - from the renin-angiotensin system to gut microbiota and malnutrition. Microbes Infect 2013;15(13):866-873.

16. Nikolaus S, Schulte B, Al-Massad N, et al. Increased tryptophan metabolism is associated with activity of inflammatory bowel diseases. Gastroenterology 2017;153(6): 1504-1516.

17. Effenberger M, Grabherr F, Mayr L, et al. Faecal calprotectin indicates intestinal inflammation in COVID-19. Gut 2020;69(8):1543-1544.

18. Beyerstedt S, Casaro EB, Rangel ÉB. COVID-19: angiotensin-converting enzyme 2 (ACE2) expression and tissue susceptibility to SARS-CoV-2 infection. Eur J Clin Microbiol Infect Dis 2021; 2:905-919.

19. Chung MK, Karnik S, Saef J, et al. SARS-CoV-2 and ACE2: The biology and clinical data settling the ARB and ACEI controversy. EBioMedicine 2020;58. doi:10.1016/j.ebiom. 2020.102907
20. Glowacka I, Bertram S, Herzog P, et al. Differential downregulation of ACE2 by the spike proteins of severe acute respiratory syndrome coronavirus and human coronavirus NL63. J Virol 2010;84(2):1198-1205.

21. Garg M, Christensen B, Lubel JS. Gastrointestinal ACE2, COVID-19 and IBD: Opportunity in the Face of Tragedy? Gastroenterology. 2020;159(4):1623-1624.e3. doi:10.1053/ j.gastro.2020.04.051

22. Glowacka I, Bertram S, Müller MA, et al. Evidence that TMPRSS2 activates the severe acute respiratory syndrome coronavirus spike protein for membrane fusion and reduces viral control by the humoral immune response. J Virol 2011;85(9):4122-4134.

23. Zang R, Gomez Castro MF, McCune BT, et al. TMPRSS 2 and TMPRSS4 promote SARS-CoV-2 infection of human small intestinal enterocytes. Sci Immunol 2020;5(47). doi:10.1126/ sciimmunol.abc3582

24. Hoffmann M, Kleine-Weber H, Schroeder S, et al. SARS-CoV-2 cell entry depends on ACE2 and TMPRSS2 and is blocked by a clinically proven protease inhibitor. Cell 2020;181(2):271-280.

25. Schuler BA, Habermann AC, Plosa EJ, et al. Age-determined expression of priming protease TMPRSS2 and localization of SARS-CoV-2 in lung epithelium. J Clin Invest 2021; 131(1). doi:10.1172/JCI140766

26. Suárez-Fariñas $M$, Tokuyama $M$, Wei $G$, et al. Intestinal inflammation modulates the expression of ACE2 and TMPRSS2 and potentially overlaps with the pathogenesis of SARS-CoV-2-related disease. Gastroenterology 2021;160(1): 287-301.

27. Nishida $\mathrm{A}$, Inoue $\mathrm{R}$, Inatomi $\mathrm{O}$, et al. Gut microbiota in the pathogenesis of inflammatory bowel disease. Clin J Gastroenterol 2018;11(1):1-10.

28. Tamboli CP, Neut C, Desreumaux $P$, et al. Dysbiosis in inflammatory bowel disease. Gut 2004;53(1):1-4.

29. Zakerska-Banaszak O, Tomczak H, Gabryel M, et al. Dysbiosis of gut microbiota in Polish patients with ulcerative colitis: a pilot study. Sci Rep 2021;11(1):2166. doi:10.1038/ s41598-021-81628-3

30. Liu $\mathrm{S}$, Zhao $\mathrm{W}$, Lan $\mathrm{P}$, et al. The microbiome in inflammatory bowel diseases: from pathogenesis to therapy. Protein Cell 2020;12(5):331-345.

31. Yeoh YK, Zuo T, Lui GCY, et al. Gut microbiota composition reflects disease severity and dysfunctional immune responses in patients with COVID-19. Gut 2021;70(4):698-706.

32. Zuo T, Zhang F, Lui GCY, et al. Alterations in Gut Microbiota of Patients With COVID-19 During Time of Hospitalization. Gastroenterology 2020;159(3):944-955.

33. Buszko M, Nita-Lazar A, Park J-H, et al. Lessons learned: new insights on the role of cytokines in COVID-19. Nat Immunol [Internet] 2021;22(4):404-11. 